\title{
Potencial de alimentação de Chrysoperla externa (Hagen, 1861) (Neuroptera, Chrysopidae) em diferentes densidades de Uroleucon ambrosiae (Thomas, 1878) (Hemiptera, Aphididae)
}

\author{
Alexander M. Auad ${ }^{1,2}$ \\ Sérgio de Freitas ${ }^{1}$ \\ Leonardo R. Barbosa ${ }^{1}$
}

\begin{abstract}
Feeding potential of Chrysoperla externa (Hagen) (Neuroptera, Chrysopidae) in different densities of Uroleucon ambrosiae (Thomas) (Hemiptera, Aphididae). The feeding potential of $2^{\text {nd }}$ and $3^{\text {rd }}$ instar larvae of Chrysoperla externa (Hagen, 1861) in relation to different densities of 30, 40 and 50 nymphs of Uroleucon ambrosiae (Thomas, 1878) at $3^{\text {rd }}$ and $4^{\text {th }}$ instars was evaluated. The treatments were individualized into $2.5 \mathrm{~cm}$ in diameter and $8.5 \mathrm{~cm}$ tall flat bottom glass vials and maintained in a controlled environmental chamber at $25 \pm 2{ }^{\circ} \mathrm{C}$ temperature, $70 \pm 10 \% \mathrm{RH}$ and $14 \mathrm{~h}$ photophase. A completely randomized experimental design with 10 replications was used. The consumption of the prey nymphs by the predator larvae was evaluated after 1, 2, 4, 8, 16 and $24 \mathrm{~h}$ from the beginning of the experiment and at every subsequent $24 \mathrm{~h}$ period until $2^{\text {nd }}$ instar larvae molted or $3^{\text {rd }}$ instar larvae pupated. Results have shown that for $2^{\text {nd }}$ instar larvae, during the $1 \mathrm{~h}$ to $24 \mathrm{~h}$ period, there was a decreasing prey consumption at the 30 and 40 prey densities. However an increase in the consumption at the 50 prey density was observed. After this period, C. externa larvae presented a progressive increase on nymphs consumption as a function of the prey density. The same occurred with de $3^{\text {rd }}$ instar predator larvae in all treatments. When daily mean consumption was evaluated the predator/prey ratio was 1:23, 1:27 and 1:33 for $2^{\text {nd }}$ instar larvae and 1:27, 1:33 and 1:41 for $3^{\text {rd }}$ instar larvae at 30,40 and 50 nymph densities, respectively.
\end{abstract}

KEYWORDS. Aphids; biological control; green lacewing; predation.

\section{INTRODUÇÃO}

Uroleucon ambrosiae (Thomas, 1878) (Hemiptera, Aphididae) alimenta-se de numerosas plantas cultivadas e silvestres da família Asteraceae, e ocasionalmente de Poaceae, causando sérios danos. Entre as hortaliças, a alface é a principal hospedeira (PeÑa-Martines 1992). Esse afídeo é um importante vetor do vírus-do-mosaico-da-alface, que ocasiona o enrolamento das folhas e prejudica o desenvolvimento das plantas (SHAWKAT et al. 1982; OMAR et al. 1985) além de acarretar danos qualitativos através da deposição de honeydew que favorece o desenvolvimento de fumagina causando desvalorização comercial do produto.

Para o controle populacional de pulgões em alface são utilizados inseticidas, fator preocupante para o consumidor, por se tratar de hortaliça consumida in natura. Dessa forma, a possibilidade do uso e o crescente aumento na comercialização de agentes de controle biológico constitui fator primordial para o manejo integrado de afídeos.

Os crisopídeos são considerados importantes predadores, podendo ser manipulados para prover o controle de afídeos em diversas culturas (ONCUER et al. 1994; GRASSwITZ \& BURTS 1995; Osman \& Selman 1996). Sucesso no controle dessa praga em alface foi obtido com liberações de Chrysoperla carnea (Stephens, 1836) (Neuroptera, Chrysopidae), em casa de vegetação, utilizando a relação predador:presa de 1:30 (Beglyarov \& Ushchekov 1977), ou quando os ovos deste predador foram colocados nas plantas de alface ( 25 a 30 ovos/ $\mathrm{m}^{2}$ ) antes do transplantio, seguindo-se três liberações semanais (QuENTIN et al. 1995). Em condições de campo houve redução de $83-88 \%$ e $90 \%$ da população de afídeos, nessa cultura, quando foram liberadas larvas de $1^{\circ}$ e $2^{\circ}$ ínstares, respectivamente (Rossmann \& ForTMAnN 1989). Dentre os crisopídeos de ocorrência no Brasil, Chrysoperla externa (Hagen, 1861), tem sido considerada de alto potencial para o controle biológico (Albuquerque et al. 1994; TAuber et al.

\footnotetext{
1. Departamento de Entomologia, Faculdade de Ciências Agrárias e Veterinárias, Universidade Estadual Paulista. 14870-000 Jaboticabal-SP, Brasil.

2. Endereço eletrônico: amauad@zipmail.com.br
} 
2000).

Esse estudo teve como objetivo avaliar o potencial de alimentação de larvas de C. externa alimentadas com diferentes densidades de ninfas de $U$. ambrosiae, em laboratório, para obtenção de informações sobre a interação predador x presa, para subsidiar programas de manejo integrado desse insetopraga em cultivo protegido de alface.

\section{MATERIALE MÉTODOS}

Manutenção das plantas de alface em cultivo hidropônico. O cultivo foi realizado em casa-de-vegetação do Departamento de Fitossanidade da Faculdade de Ciências Agrárias e Veterinárias-UNESP, Jaboticabal, SP.

Semanalmente, 80 mudas de alface cv. Verônica foram plantadas em bandejas e após vinte dias transplantadas de forma a manter cinco lotes de plantas com diferentes idades.

Diariamente, o nível do reservatório de água (capacidade para $500 \mathrm{~L}$ ) foi verificado e restaurado e a necessidade de reposição dos nutrientes foi avaliada por meio de um condutivímetro. Este aparelho fornece informações sobre a concentração de sais da solução nutritiva, sem informar as concentrações individuais dos nutrientes, realizando-se a reposição toda vez que o valor foi inferior a $2 \mathrm{mS} / \mathrm{cm}$ (milisiemens), conforme recomendações da NFT (Nutrient Film Technique). A solução nutritiva foi totalmente reposta a cada 15 dias, seguindo as recomendações de FURLANI (1995), para os nutrientes utilizados e suas respectivas doses.

Criação de manutenção de $\boldsymbol{U}$. ambrosiae. Ninfas e adultos foram coletados em plantas de alface em cultivo hidropônico, no Colégio Técnico Agrícola da Universidade Estadual Paulista (UNESP), Jaboticabal, SP, e transferidos para as plantas de alface na estufa de manutenção, efetuando-se, diariamente, a observação e eliminação de inimigos naturais.

Criação de manutenção de $C$. externa. Adultos do crisopídeo foram coletados com auxílio de rede entomológica em gramíneas, próximas a um bosque de Pinus sp., no Campus da UNESP-Jaboticabal, SP, e levados para o Laboratório de Biossistemática e Criação Massal de Crisopídeos do
Departamento de Fitossanidade, onde procedeu-se à identificação de C. externa.

Os adultos foram mantidos em gaiolas cilíndricas de PVC ( $23 \mathrm{~cm}$ de diâmetro $\mathrm{x} 10 \mathrm{~cm}$ de altura) vedadas na parte inferior com tecido de nylon fixado com anéis de $1 \mathrm{~cm}$ de largura e diâmetro menor que o da gaiola, de modo a ajustarem-se na borda interna do tubo. A extremidade superior também foi vedada com nylon, fixado com goma elástica. As gaiolas foram revestidas internamente com papel sulfite branco e a dieta foi constituída de lêvedo de cerveja e mel na proporção de 1:1.

Os ovos de C. externa foram coletados diariamente colocados na densidade de 20 por recipiente plástico $(5,5 \mathrm{~cm}$ de diâmetro x $8 \mathrm{~cm}$ de altura). As larvas foram alimentadas com ovos de Sitotroga cerealella (Oliver, 1819) (Lepidoptera, Gelechiidae), colados com solução de goma arábica e água (1:1) em seções de cartolina preta de $2,5 \mathrm{~cm}^{2}, 12,5 \mathrm{~cm}^{2}$ e 18,8 $\mathrm{cm}^{2}$, e colocados à disposição para as larvas de $1^{\circ}$, $2^{\circ}$ e $3^{\circ}$ ínstares, respectivamente. Os adultos foram colocados no mesmo tipo de gaiola, descrito anteriormente, para a obtenção de ovos (geração $\mathrm{F}_{2}$ ) a serem usados no experimento.

Instalação do experimento. Ninfas de $3^{\circ}$ e $4^{\circ}$ ínstares de $U$. ambrosiae foram oferecidas para larvas de $2^{\circ}$ ou $3^{\circ}$ ínstar do crisopídeo previamente alimentadas com ovos de $S$. cerealella. Utilizaram-se as densidades de 30, 40 e 50 ninfas por recipiente de criação $(2,5 \mathrm{~cm} \times 8,5 \mathrm{~cm})$, os quais foram vedados com filme de polietileno e mantidos a $25+2{ }^{\circ} \mathrm{C}, 70+10 \%$ UR e fotofase de 14 horas.

Avaliou-se o número de afídeos sobreviventes e aqueles que aparentemente morreram de forma natural, subtraindo-os da densidade inicial, após $1,2,4,8,16$ e 24 h do início do experimento e, posteriormente a cada $24 \mathrm{~h}$, até a mudança de ínstar ou a pupação. Após cada avaliação, os afídeos foram removidos e substituídos, mantendo-se a densidade inicial. Utilizou-se o delineamento inteiramente casualizado, com 10 repetições. Foi feita análise de variância e as médias foram comparadas pelo teste de Tukey $(\mathrm{P}<0,05)$.

\section{RESULTADOSEDISCUSSÃO}

Não houve diferença na capacidade predatória de larvas de

Tabela 1. Consumo acumulado e médio diário $( \pm \mathrm{EP})$ de ninfas de $3^{\circ}$ e $4^{\circ}$ ínstares de $U$. ambrosiae, fornecidos em três densidades, por larvas de $2^{\circ}$ e $3^{\circ}$ ínstares de $C$. externa. Temperatura $25 \pm 2{ }^{\circ} \mathrm{C}, 70 \pm 10 \%$ UR e 14 horas de fotofase.

\begin{tabular}{|c|c|c|c|c|c|c|c|c|c|c|}
\hline \multicolumn{11}{|c|}{ Horas após início do teste } \\
\hline & 1 & 2 & 4 & 8 & 16 & 24 & 48 & 72 & 96 & $\begin{array}{l}\text { Médio } \\
\text { diário }\end{array}$ \\
\hline Densidade & \multicolumn{10}{|c|}{ Larvas de $2^{\circ}$ ínstar } \\
\hline 30 & $5,5 \pm 0,56 \mathrm{a}$ & $8,6 \pm 0,54 a$ & $10,9 \pm 0,82 \mathrm{a}$ & $15,4 \pm 1,02 \mathrm{a}$ & $24,5 \pm 1,02 \mathrm{ab}$ & $32,5 \pm 1,19 b$ & $49,1 \pm 1,08 b$ & $68,5 \pm 1,96 \mathrm{c}$ & - & $22,8 \pm 0,65 \mathrm{c}$ \\
\hline 40 & $4,0 \pm 0,71 \mathrm{a}$ & $7,3 \pm 0,96 \mathrm{a}$ & $9,4 \pm 1,08 \mathrm{a}$ & $13,9 \pm 1,23 \mathrm{a}$ & $22,0 \pm 1,95 \quad b$ & $29,7 \pm 2,42 b$ & $51,2 \pm 2,37 b$ & $81,7 \pm 2,30 \mathrm{~b}$ & - & $27,3 \pm 0,77 b$ \\
\hline 50 & $6,0 \pm 0,63 \mathrm{a}$ & $8,5 \pm 0,37 \mathrm{a}$ & $10,7 \pm 0,43 \mathrm{a}$ & $17,0 \pm 0,94 \mathrm{a}$ & $27,9 \pm 1,54 \mathrm{a}$ & $40,8 \pm 2,48 \mathrm{a}$ & $65,5 \pm 3,45 \mathrm{a}$ & $97,6 \pm 2,84 \mathrm{a}$ & - & $32,6 \pm 0,95 \mathrm{a}$ \\
\hline \multicolumn{11}{|c|}{ Larvas de $3^{\circ}$ ínstar } \\
\hline 30 & $2,5 \pm 0,27 \mathrm{a}$ & $5,1 \pm 0,38 \mathrm{a}$ & $7,4 \pm 0,60 \quad b$ & $12,4 \pm 0,95 \quad b$ & $22,7 \pm 1,18 \quad b$ & $33,5 \pm 2,08 \mathrm{~b}$ & $59,3 \pm 2,19 b$ & $87,5 \pm 2,17 \mathrm{c}$ & $106,8 \pm 2,30 \mathrm{c}$ & $26,7 \pm 0,57 \mathrm{c}$ \\
\hline 40 & $3,5 \pm 0,31 \mathrm{a}$ & $6,3 \pm 0,47 \mathrm{a}$ & $9,4 \pm 0,70 \mathrm{ab}$ & $14,4 \pm 1,11 \mathrm{ab}$ & $24,0 \pm 1,14 \mathrm{ab}$ & $37,0 \pm 1,74 a b$ & $68,9 \pm 3,92 b$ & $105,4 \pm 3,97 b$ & $133,8 \pm 3,94 b$ & $33,5 \pm 0,98 b$ \\
\hline 50 & $3,7 \pm 0,47 \mathrm{a}$ & $6,4 \pm 0,45 \mathrm{a}$ & $9,6 \pm 0,48 \mathrm{a}$ & $15,9 \pm 0,91 \mathrm{a}$ & $27,6 \pm 1,11 \mathrm{a}$ & $40,9 \pm 1,57 \mathrm{a}$ & $85,3 \pm 1,58 \mathrm{a}$ & $129,3 \pm 2,11 \mathrm{a}$ & $164,3 \pm 2,78 \mathrm{a}$ & $41,3 \pm 0,75 \mathrm{a}$ \\
\hline
\end{tabular}

Médias seguidas por letras distintas nas colunas diferem entre si pelo teste de Tukey $(\mathrm{P}<0,05)$ 
Tabela 2. Consumo acumulado médio $( \pm \mathrm{EP})$ de ninfas de $3^{\circ}$ e $4^{\circ}$ ínstares de $U$. ambrosiae, por larvas de $2^{\circ}$ e $3^{\circ}$ ínstares de $C$. externa. Temperatura $25 \pm 2{ }^{\circ} \mathrm{C}, 70 \pm 10 \%$ UR e 14 horas de fotofase.

\begin{tabular}{|c|c|c|c|c|c|c|c|c|}
\hline \multicolumn{9}{|c|}{ Horas após início do teste } \\
\hline Estádio & 1 & 2 & 4 & 8 & 16 & 24 & 48 & 72 \\
\hline Segundo ínstar & $5,2 \pm 0,39 a$ & $8,2 \pm 0,39 \mathrm{a}$ & $10,7 \pm 0,50 \mathrm{a}$ & $5,4 \pm 0,64 \mathrm{a}$ & $24,8 \pm 0,97 \mathrm{a}$ & $34,3 \pm 1,47 \mathrm{a}$ & $55,3 \pm 1,94 b$ & $82,6 \pm 2,58 \mathrm{~b}$ \\
\hline Terceiro ínstar & $3,2 \pm 0,22 b$ & $5,9 \pm 0,27 b$ & $8,8 \pm 0,38 b$ & $14,2 \pm 0,61 \mathrm{a}$ & $24,8 \pm 0,74 \mathrm{a}$ & $37,1 \pm 1,15 \mathrm{a}$ & $71,2 \pm 2,51 \mathrm{a}$ & $107,5 \pm 3,56$ a \\
\hline
\end{tabular}

Médias seguidas por letras distintas nas colunas diferem entre si pelo teste de Tukey $(\mathrm{P}<0,05)$.

segundo ínstar de C. externa, em função das densidades de afídeos, até 8 horas após o fornecimento da presa. A partir de 16 horas, houve um maior consumo por larvas mantidas na densidade de 50 presas, não sendo constatadas diferenças significativas entre as menores densidades. Porém, após 72 horas, larvas supridas com a menor densidade predaram um número significativamente menor de pulgões (Tabela 1). Estes resultados concordam com aqueles obtidos por SoLOMON (1969) que demonstrou que as oportunidades de encontro são favorecidas em maiores densidades da presa, e com os de Hodek (1967) que mencionou que, em densidades populacionais mais elevadas, os predadores da família Coccinellidae, necessitam de menos tempo para alimentaremse da presa, acarretando em um maior consumo.

Para as larvas de terceiro ínstar não houve efeito da densidade da presa sobre a capacidade predatória nas duas primeiras horas de avaliação. Entre 4 e 48 horas, o consumo pelas larvas que receberam as densidades de 30 e 40 presas não diferiu significativamente e foi inferior àquelas larvas alimentadas com 50 ninfas. Contudo, nas avaliações realizadas após 72 e 96 horas, o número de afídeos consumidos foi menor na densidade de 30 ninfas, apresentando um aumento significativo com o acréscimo na densidade de presas disponível (Tabela 1). Esses resultados assemelham-se aos de NordLund \& MorRISON (1990), que demostraram um maior consumo por Chrysoperla rufilabris (Burmeister, 1839) com o aumento do número de Aphis gossypii Glover,1877 ou ovos e lagartas de $1^{\circ}$ ínstar de Heliothis virescens (Fabricius, 1781); com os de KABISSA et al. (1996) para larvas dos crisopídeos Mallada desjardinsi (Navás, 1911) e Chrysoperla congrua (Walker, 1853), alimentadas com o pulgão A. gossypii nas densidades de 5, 10, 15, 20 e 25 indivíduos; e também aos de YuKsel \& Gocmem (1992), para larvas de C. carnea alimentadas com A. gossypii.

Nas primeiras 4 horas de avaliação, observou-se menor consumo por larvas de terceiro ínstar em relação àquelas de segundo, independente da densidade. Entre 8 e 24 horas, o número de presas consumidas foi semelhante para ambos os estádios, e após esse período, constatou-se um maior consumo por larvas de terceiro ínstar (Tabela 2). Isto pode ser atribuído ao maior tempo requerido pelas larvas de terceiro ínstar para se adaptarem ao novo tipo de presa, uma vez que, durante os estádios iniciais, se alimentaram de ovos de $S$. cerealella. AUAD et al. (2001) verificaram que larvas de terceiro ínstar previamente alimentadas com ovos dessa espécie tiveram um consumo de
U. ambrosiae superior às do mesmo ínstar que sempre alimentaram-se do afídeo, denotando influência do regime alimentar na capacidade de predação de C. externa.

Avaliando-se a capacidade diária de predação por larvas de segundo ínstar, verificou-se um aumento no número de presas consumidas em função do acréscimo na densidade de presas disponíveis, obtendo-se a relação predador-presa de $1: 23 ; 1: 27$ e $1: 33$ nas densidades 30,40 e 50 ninfas, respectivamente (Tabela 1). Para aquelas de terceiro ínstar, a taxa de consumo médio diário foi superior à encontrada para as de segundo ínstar e a relação predador:presa variou com a densidade utilizada, correspondendo a 1:27, 1:33 e 1:41 nas densidades de 30, 40 e 50 afídeos, respectivamente, sendo estes valores inferiores àqueles obtidos por SCOPEs (1969), que encontrou uma relação de uma larva de $3^{\mathcal{Q}}$ ínstar de $C$. carnea para 200 M. persicae, em crisântemo, em casa-de-vegetação.

A variação na eficiência de controle de C. carnea, de acordo com a densidade de afídeos, foi demonstrada por Scopes (1969), Beglyarov \& Ushchekov (1977) e Oncuer et al. (1994); no entanto, para C. externa, pesquisas dessa natureza realizadas com U. ambrosiae, são inexistentes. Face às respostas desse predador, considera-se que a sua utilização no controle desse inseto-praga, na cultura da alface, em cultivo hidropônico, denota ser eficiente. As interações encontradas podem indicar o número de predadores que devem ser testados em programas de controle biológico de $U$. ambrosiae por larvas de segundo e terceiro ínstares de C. externa, em casa-de-vegetação.

Agradecimentos. À Fundação de Amparo à Pesquisa do Estado de São Paulo, FAPESP, pelo auxílio financeiro para execução do trabalho.

\section{REFERÊNCIAS}

Albuquerque, G. S.; C. A. Tauber \& M. J. Tauber. 1994. Chrysoperla externa (Neuroptera: Chrysopidae): Life history and potential for biological control in Central and South América. Biological Control 4(2): 8-13.

Auad, A. M.; S. Freitas \& L. R. Barbosa. 2001. Influencia de la dieta en la respuesta funcional de Chrysoperla externa (Hagen) (Neuroptera: Chrysopidae) alimentadas con Uroleucon ambrosiae (Thomas) (Hemiptera: Aphididae). Boletín de Sanidad Vegetal Plagas 27(4): 455-463.

Beglyarov, G. A. \& A. T. UshcheKov. 1977. Biological control of aphids on green crop. Zashchita Rastenii 2: 25-27.

Furlani, P. R. 1995. Cultivo de alface pela técnica de hidroponiaNFT. Campinas, Instituto Agronômico (Documento IAC,55), 18 p. Grasswitz, T. R. \& E. C. Burts. 1995. Effect of native natural enemies 
and augmentative releases of Chrysoperla rufilabris Burmeister and Aphidoletes aphidimyza (Rondani) on the population dynamics of the green apple aphid, Aphis pomi DeGeer. International Journal of Pest Management 41(3): 176-183.

Hodek, I. 1967. Bionomics and ecology of predaceous Coccinellidae. Annual Review of Entomology 12: 79-104.

Kabissa, J. C. B.; J. G. Yarro; H. Y. Kayumbo \& S. A. Juliano. 1996. Functional response of two chrysopid predators feeding on Helicoverpa armigera (Lep.: Noctuidae) and Aphis gossypii (Hom.: Aphididae). Entomophaga 41(2): 141-151.

Nordlund, D. A. \& R. K. Morrison. 1990. Handling time, prey preference, and functional response for Chrysoperla rufilabris in the laboratory. Entomologia Experimentalis et Applicata 57: 237-242.

Omar, R. A.; F. F. Mehiar; E. A. Zayed \& A. A. Deif. 1985. Biological studies on some seed-borne viruses and their effect on vegetative growth and yield component of the host plants. Acta Physiologica Academiae Scientiarum Hungaricae 20(1/2): 59-78.

Oncuer, C.; Z. Yoldas; N. Madanlar \& A. Gul. 1994. Biological control of insect pests on greenhouse vegetables in the Aegean Region. Mucadele Kongresi Bildirileeri, 3, 1994, Izmir, Eg e Universitesi Ziraat Fakultesi, Bitki Koruma Bolumu, Izmir, p. 395-407.

Osman, M. Z. \& B. J. Selman. 1996. Effect of larval diet on the performance of the predator Chrysoperla carnea Stephens (Neuropt., Chrysopidae). Journal of Applied Entomology 120(2): 115-117.

PeÑa-Martines, R. 1992. Identificação de afidos de importancia agricola. In: M. C. Urias; M. R. Rodriguez \& A. T. Alejandre (eds.). Afidos como vetores de vírus en México. México, Centro de Fitopatologia, 1: $135 \mathrm{p}$.

Quentin, U.; M. Hommes \& T. Basedow. 1995. Studies on the biological control of aphids (Hom., Aphididae) on lettuce in greenhouse. Journal of Applied Entomology 119(3): 227-232.

Rossmann, F. \& M. Fortmann. 1989. Investigations on the use of the predator Chrysoperla carnea Steph. (Neuroptera, Chrysopidae) for the control of aphids in the garden. Mitteilngen de Deutschen Gesellschaft pelle Allgemeine und Angewandte Entomoligie 7(1/3): 295-297.

SCopes, N. E. A. 1969. The potential of Chrysopa carnea as a biological control agent of Myzus persicae on glasshouse chrysanthemums. Annals of Applied Biology 64: 433-489.

Shawkat, A. L. B.; G. L. Fegla \& N. A. Ramadan. 1982. Occurrence of lettuce mosaic virus on lettuce in Iraq. Mesopotamia. Journal of Agriculture 17(1): 79-93.

Solomon, M. E. 1969. The natural control of animal populations. Journal of Animal Ecology 18: 1-35.

Tauber, M. J.; C. A. Tauber; K. M. Dahne \& K. S. Hagen. 2000. Commercialization of predator: Recent lessons from green lacewings (Neuroptera: Chrysopidae: Chrysoperla). American Entomology 46(1): 26-34.

Yunsel, S. \& H. Gocmen. 1992. The effectiveness of Chrysoperla carnea (Stephens) (Neuroptera, Chrysopidae) as a predator on cotton aphid Aphis gossypii Glov. (Homoptera, Aphididae). Proceeding Turkish National Congress of Entomology 2: 209-216. 